\title{
Propuesta de innovación docente en la asignatura Empresas y Organizaciones Turísticas
}

\section{Teaching innovation proposal in the subject Tourism Companies and Organizations}

Paula Villalba Ríos

ORCID: https://orcid.org/0000-0002-7488-6110

Universidad de Sevilla

Departamento de Administración de Empresas y Marketing

pvillalba@us.es

DOI: http://dx.doi.org/10.12795/9788447231003.072

Pp.: 1531-1552 


\section{Contexto de la asignatura}

La enseñanza universitaria, de un tiempo a esta parte, se está enfrentando a múltiples y diversos retos, como son aquellos relacionados con la implementación del Espacio Europeo de Educación Superior (EEES) o, más recientemente, los derivados de la pandemia causada por el SARSCOV-2. La receta ante estos desafíos pasa por situar al alumnado en el núcleo del proceso de enseñanza-aprendizaje de manera que sean ellos quienes tomen el control de su propia formación (Finkel, 2000). Así, el uso de un modelo metodológico de carácter constructivista (Porlán, 2020) puede ayudar en la consecución de este objetivo.

Particularmente, en el área de turismo y finanzas, son varios los ciclos de innovación y mejora en el aula (en adelante CIMA) que se han realizado haciendo uso del citado modelo metodológico y buscando este rol activo del alumnado (Pedregoso-Mateos, 2018; Zerva, 2020) en el marco del programa Formación e Innovación Docente del Profesorado (FIDOP) que desarrolla desde hace unos años la Universidad de Sevilla. Por ejemplo, Rosa-Díaz (2020) propone una serie de actividades tanto individuales como en equipo, como son los estudios de casos, los debates o la presentación de informes. Estos CIMAs me han servido como guía y apoyo a esta experimentación.

La asignatura donde he diseñado y desarrollado el CIMA es Empresas y Organizaciones Turísticas. Se trata de una asignatura de formación básica (de 6 créditos) de primer curso del Grado en Turismo que pretende introducir al alumno o alumna en los principales conceptos y temas empresariales, así como, profundizar en aspectos concretos de la gestión turística, como las formas de competir y cooperar tradicionales en el sector.

Ciclos de Mejora en el Aula (2020). Experiencias de Innovación Docente de la US Esta obra se distribuye con la licencia Creative Commons 
Cuatro profesores, en el presente curso 2020/2021, tenemos docencia en los cinco grupos de en torno a 80 estudiantes de esta asignatura. En mi caso, soy la profesora de dos de los cinco grupos, ambos en turno de tarde. En concreto, los alumnos y alumnas con quienes he experimentado este CIMA son los que pertenecen al subgrupo 2 del grupo 2. Cabe señalar que, de las 38 matriculaciones, 19 han cursado anteriormente la asignatura. El horario de clase es de 16:00 a 18:00, lunes y miércoles.

Para este año académico inusual se han diseñado cuatro tipos de sesiones. Para entender la lógica que se usa en el diseño de este sistema, es necesario tener en cuenta que los estudiantes, según la planificación, asisten día sí, día no a la facultad, por lo que cada subgrupo está presencialmente en la asignatura en semanas alternas. De esta forma, se desarrollan las sesiones presenciales, son clases teórico/prácticas donde se comienza con la corrección de un caso previamente trabajado y se avanza en los contenidos de la asignatura. Por otro lado, de forma virtual, los alumnos y alumnas trabajan, en grupos, mediante la herramienta Blackboard Collaborate Ultra, los casos que se debaten en las sesiones que se describen previamente. Adicionalmente, existen otros tipos de sesiones: (i) sesiones de resolución de casos trabajados en casa y, (ii) sesiones de trabajo en grupo, donde trabajan en un proyecto.

Este curso es el segundo año que tengo asignada docencia, sin embargo, repito asignatura, por lo que tengo una breve experiencia en la materia. Considero que se trata de una asignatura muy bien estructurada, donde los contenidos a trabajar y los objetivos propuestos están claros. Además, es una grata experiencia formar parte de una de las primeras experiencias del alumnado en su paso por la universidad.

Ciclos de Mejora en el Aula (2020). Experiencias de Innovación Docente de la US Esta obra se distribuye con la licencia Creative Commons 
El tema 2, "La organización turística como sistema", y parte del 3, "El entorno de la empresa turística", se proponen para la aplicación del CIMA que ha ocupado tres sesiones y media de dos horas, sumando siete horas de docencia en total. Estos temas son básicos y esenciales en el programa de la asignatura, ya que sirven como cimientos para la comprensión de los temas consecutivos con contenidos más complejos.

\section{Diseño del ciclo de mejora en el aula}

El presente apartado comienza con la presentación del mapa de contenidos, posteriormente describe del modelo metodológico posible y, por último, recoge la secuencia de actividades desarrollada durante el CIMA.

\section{Mapa de contenidos}

El mapa de contenidos (figura 1) se ha diseñado para el tema 2 "La organización turística como sistema" y parte del tema 3 "El entorno de la empresa turística" porque están estrechamente relacionados. El tema 2 versa sobre el funcionamiento interno de la empresa turística que se aborda a través de la teoría de sistemas, mientras que la primera parte del tema 3 trata sobre las características y elementos del entorno general y específico de la empresa turística (Casanueva y Gallego, 2016).

En el mapa de contenidos se pueden observar (i) contenidos de carácter teórico señalados en rosa; contenidos de carácter procedimental, en verde; y (iii) contenidos actitudinales, en azul. Asimismo, las palabras en mayúsculas indican conceptos troncales y la forma de las figuras la jerarquía que se establece entre ellos. Por último, las líneas continuas indican una relación directa entre los contenidos.

Ciclos de Mejora en el Aula (2020). Experiencias de Innovación Docente de la US Esta obra se distribuye con la licencia Creative Commons 
Los alumnos y las alumnas ya conocen qué es una empresa, de qué elementos se compone, qué funciones realiza y algunas particularidades de las empresas turísticas. Partiendo de ahí, el mapa de contenidos trata de mostrar que aquello que ocurre en el interior de las organizaciones no son actividades aisladas, sino que están relacionadas entre sí y, al mismo tiempo, abiertas a la influencia de agentes externos. Con ese fin, consideramos que las empresas son sistemas que podemos dividir en subsistemas, entre los que proponemos algunos que están presentes en la mayoría de estas. Observando el mapa podemos percatarnos de que estos subsistemas se encuentran estrechamente relacionados entre sí y con el exterior. Como vemos, el entorno influye y puede influir en las empresas, por ello es necesario conocer sus características y delimitar su amplitud (entorno general y específico).

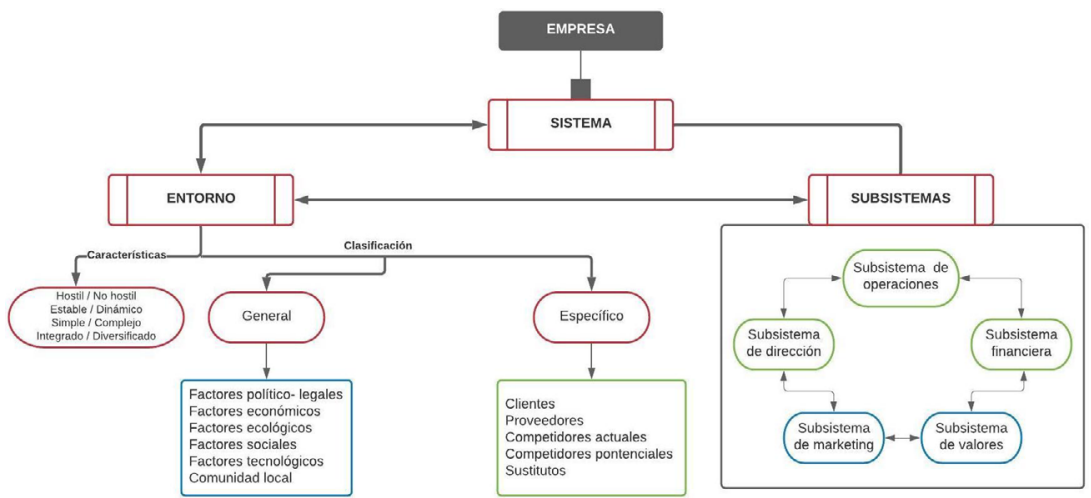

Figura 1. Mapa conceptual

\section{Modelo metodológico}

En el modelo que venía utilizando, los estudiantes asistían a las clases presenciales con las respuestas que, en sesiones virtuales de trabajo en grupo, habian dado a diferentes casos de estudio. En clase, revisamos y debatimos estos casos y tratamos los contenidos mediante clases magistrales participativas.

Ciclos de Mejora en el Aula (2020). Experiencias de Innovación Docente de la US Esta obra se distribuye con la licencia Creative Commons 
Con el diseño de un nuevo modelo metodológico pretendo lograr un cambio significativo en la secuencia de actividades, que diseño para el desarrollo de este CIMA, que dé lugar a una mejora en el proceso de enseñanza-aprendizaje. En primer lugar, planteo al alumnado un problema/ cuestión a resolver $(P)$, que da lugar a la explicitación de sus ideas (EI), es decir, qué sabemos ya. Posteriormente, realizamos actividades de contraste (AC) y de recapitulación para confrontar los nuevos aportes de conocimiento con el punto de partida (AR) y por último llegan por ellos mismos a una conclusión (C) que les permite responder a la pregunta inicial.

Considero que la enseñanza que se adapta a este modelo metodológico va a aumentar el aprendizaje de los estudiantes ya que (i) partir de un problema los engancha y motiva, (ii) conocer el punto de partida me permite modular y moldear el discurso y personalizarlo, (iii) los alumnos y alumnas hacen explicitas sus ideas y esto les permite ubicarse en el tema $y$, posteriormente (iv) ponerlas en cuestión, lo que les lleva a elaborar explicaciones más completas y complejas, siendo conscientes de esta evolución y, por último, (v) establecer una conclusión nos ayuda a cerrar el ciclo (De Alba y Porlán, 2017).

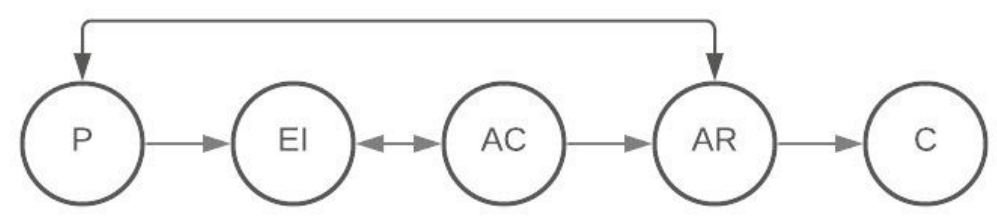

Figura 2. Modelo metodológico posible

Con el modelo metodológico propuesto (figura 2), pretendo pasar de un modelo metodológico más transmisivo a uno más investigativo y constructivista (Porlán, 2020) que

Ciclos de Mejora en el Aula (2020). Experiencias de Innovación Docente de la US Esta obra se distribuye con la licencia Creative Commons 
ponga el foco, siempre, en el alumnado como sujeto activo en el proceso de enseñanza- aprendizaje y me asigne como docente un rol de facilitadora del aprendizaje significativo de estos (Finkel, 2000).

\section{Secuencia de actividades del CIMA}

En la siguiente tabla muestro la secuencia de actividades que ha conformado este CIMA. El diseño de esta secuencia lo he realizado a partir del modelo metodológico anteriormente indicado. Además, ha sido elaborada de manera que ayude a conseguir el objetivo de crear un entorno para el aprendizaje crítico natural, conseguir y no perder la atención del alumno o alumna, crear diferentes experiencias de aprendizaje y ayudar a que los estudiantes aprendan fuera de clase, tal y como propone Bain (2004).

En la tabla reflejo las actividades, su descripción y el momento del modelo metodológico al que corresponden, así como el tiempo real de su realización. Además, se incluyen los problemas que hemos abordado y los contenidos a los que hacen referencia. Estos problemas, han constituido las preguntas del cuestionario que se describe posteriormente y han sido de suma utilidad para la explicitación de las ideas previas de los estudiantes.

Tabla 1. Secuencia de actividades.

\begin{tabular}{|l|l|l|}
\hline \multicolumn{3}{|c|}{ Sesión 1 } \\
\hline \multicolumn{3}{|c|}{$\begin{array}{c}\text { Problema 1. ¿Sería importante para ti conocer internamente la empresa } \\
\text { que diriges o para la que trabajas? ¿Por qué? ¿Cómo se te ocurre que } \\
\text { puedes analizarla? }\end{array}$} \\
\multicolumn{2}{|c|}{ Contenido. La empresa como sistema. } & Tiempo \\
\hline Actividad & Descripción & 10 min \\
\hline 1 & $\begin{array}{l}\text { Presentación } \\
\text { del problema + } \\
\text { Ideas previas. } \\
\text { P+El. }\end{array}$ & $\begin{array}{l}\text { Reflexionar individualmente sobre las } \\
\text { preguntas planteadas. Escribir en la laferentes respuestas dadas y } \\
\text { debatir. }\end{array}$ \\
\hline
\end{tabular}

Ciclos de Mejora en el Aula (2020). Experiencias de Innovación Docente de la US Esta obra se distribuye con la licencia Creative Commons 


\begin{tabular}{|c|c|c|c|}
\hline 2 & $\begin{array}{l}\text { Explicación } \\
\text { teórica } \\
\text { participativa. } \mathbf{A C} \text {. }\end{array}$ & $\begin{array}{l}\text { Reorganizar, modular y corregir ideas } \\
\text { previas, explicar qué es y la utilidad de } \\
\text { la Teoría de Sistemas en el análisis de } \\
\text { la empresa }\end{array}$ & $20 \mathrm{~min}$ \\
\hline 3 & $\begin{array}{l}\text { Caso de estudio } \\
\text { Disneyland. AC. }\end{array}$ & $\begin{array}{l}\text { Exponer soluciones a las preguntas del } \\
\text { caso Disneyland previamente trabajado. } \\
\text { Construir un mapa de respuestas. }\end{array}$ & $20 \min$ \\
\hline 4 & Juego de rol. $\mathbf{A C}$. & $\begin{array}{l}\text { Proyectar una problemática dada en } \\
\text { Disneyland. Definir roles. Representar } \\
\text { la situación. }\end{array}$ & $10 \mathrm{~min}$ \\
\hline 5 & $\begin{array}{l}\text { Conclusión } \\
\text { sobre la teoría } \\
\text { de sistemas. C. }\end{array}$ & $\begin{array}{l}\text { Proyectar el mapa de contenidos } \\
\text { identificar dónde nos encontramos } \\
\text { y preguntar hacia dónde creen que } \\
\text { vamos. }\end{array}$ & $5 \mathrm{~min}$ \\
\hline \multicolumn{4}{|c|}{ Sesión 2} \\
\hline \multicolumn{4}{|c|}{$\begin{array}{l}\text { Problema 2. Si tuviésemos que descomponer a Ryanair en subsistemas } \\
\text { ¿Qué separación propones? ¿De qué se encargaría cada uno de ellos? } \\
\text { Contenido. Los subsistemas de las empresas y organizaciones turísticas. }\end{array}$} \\
\hline \multicolumn{2}{|c|}{ Actividad } & Descripción & Tiempo \\
\hline 6 & $\begin{array}{l}\text { Resumen sesión } \\
1+\text { Ideas previas } \\
\text { plasmadas en el } \\
\text { test inicial. P+El. }\end{array}$ & $\begin{array}{l}\text { Retomar ideas del día anterior y } \\
\text { proyectar respuestas dadas a esta } \\
\text { pregunta en el test inicial. Preguntar, } \\
\text { ¿sería esta clasificación aplicable a } \\
\text { Disneyland? ¿Por qué? }\end{array}$ & $25 \mathrm{~min}$ \\
\hline 7 & $\begin{array}{l}\text { Señalización } \\
\text { del camino a } \\
\text { recorrer. AC. }\end{array}$ & $\begin{array}{l}\text { Proyectar el mapa de contenidos y } \\
\text { señalar al alumnado qué abordaremos } \\
\text { en la sesión y por qué a partir de sus } \\
\text { respuestas dadas. }\end{array}$ & $5 \mathrm{~min}$ \\
\hline 8 & $\begin{array}{l}\text { Caso de estudio } \\
\text { Disneyland. El. }\end{array}$ & $\begin{array}{l}\text { Debatir el caso Disneyland. Representar } \\
\text { en la pizarra las ideas principales } \\
\text { obtenidas del debate de los casos. }\end{array}$ & $10 \mathrm{~min}$ \\
\hline 9 & $\begin{array}{l}\text { Explicación } \\
\text { teórica } \\
\text { participativa. AC. }\end{array}$ & $\begin{array}{l}\text { A partir de las respuestas dadas } \\
\text { explicar contenidos sobre "Los } \\
\text { Subsistemas de la Empresa". Fomentar } \\
\text { la continua participación del } \\
\text { estudiante haciéndole preguntas. }\end{array}$ & $70 \mathrm{~min}$ \\
\hline 10 & $\begin{array}{l}\text { Caso de estudio } \\
\text { Disneyland. AR. }\end{array}$ & $\begin{array}{l}\text { Añadir, eliminar, ordenar las } \\
\text { respuestas expuestas en la actividad } \\
\text { 8. Se deja tiempo para tratar de } \\
\text { profundizar en ellas con información } \\
\text { actual. }\end{array}$ & \\
\hline
\end{tabular}

Ciclos de Mejora en el Aula (2020). Experiencias de Innovación Docente de la US Esta obra se distribuye con la licencia Creative Commons Reconocimiento-NoComercial-SinObraDerivada Internacional (CC BY-NC-ND 4.0.) 


\begin{tabular}{|c|c|c|c|}
\hline 11 & Kahoot. C. & $\begin{array}{l}\text { Kahoot sobre todo lo abordado en la } \\
\text { sesión. }\end{array}$ & $10 \mathrm{~min}$ \\
\hline \multicolumn{4}{|c|}{ Sesión 3} \\
\hline & Actividad & Descripción & Tiempo \\
\hline 12 & Mi mapa. C. & $\begin{array}{l}\text { Construir un mapa conceptual sobre } \\
\text { los contenidos vistos en relación } \\
\text { con los subsistemas de la empresa. } \\
\text { Actividad no presencial. Grupos de } \\
\text { trabajo. }\end{array}$ & $60 \mathrm{~min}$ \\
\hline 13 & $\begin{array}{l}\text { Caso de estudio. } \\
\text { El. }\end{array}$ & $\begin{array}{l}\text { ¿Cómo afecta el entorno empresarial a } \\
\text { la actividad de una empresa? Resolver } \\
\text { preguntas del caso del tema con sus } \\
\text { conocimientos actuales. Actividad no } \\
\text { presencial. Grupos de trabajo. }\end{array}$ & $60 \mathrm{~min}$ \\
\hline \multicolumn{4}{|c|}{ Sesión 4} \\
\hline \multicolumn{4}{|c|}{$\begin{array}{c}\text { Problema 3. Imaginamos que somos parte de la alta dirección de Ryanair } \\
\text { y estamos tratando de diseñar la estrategia de cara a los próximos tres } \\
\text { años. ¿Cuáles son los factores del entorno general que deberíamos tener } \\
\text { en cuenta? ¿Por qué? si miramos al entorno específico. ¿Cuáles son los } \\
\text { factores que tendríamos en cuenta? ¿Cómo podría afectar cada uno de } \\
\text { ellos a Ryanair? } \\
\text { Contenido. El entorno empresarial. }\end{array}$} \\
\hline & Actividad & Descripción & Tiempo \\
\hline 14 & $\begin{array}{l}\text { Resumen sesión } \\
3+\text { Ideas previas } \\
\text { plasmadas en } \\
\text { el test inicial. } \mathbf{P} \\
\text { + El. }\end{array}$ & $\begin{array}{l}\text { Proyectar respuestas dadas a esta } \\
\text { pregunta en el test inicial. Representar } \\
\text { las respuestas dadas en la actividad } \\
\text { 13. }\end{array}$ & $15 \mathrm{~min}$ \\
\hline
\end{tabular}

Ciclos de Mejora en el Aula (2020). Experiencias de Innovación Docente de la US Esta obra se distribuye con la licencia Creative Commons 


\begin{tabular}{|ll|l|l|}
\hline 15 & $\begin{array}{l}\text { Explicación } \\
\text { teórica } \\
\text { participativa. AC. }\end{array}$ & $\begin{array}{l}\text { Reorganizar, modular y corregir ideas } \\
\text { previas, explicar qué tener en cuenta } \\
\text { en el análisis del entorno. Diferencias } \\
\text { entre entorno general y específico. }\end{array}$ & 90 min \\
\cline { 1 - 3 } 16 & $\begin{array}{l}\text { Caso de estudio. } \\
\text { AR. }\end{array}$ & $\begin{array}{l}\text { Añadir, eliminar, ordenar las } \\
\text { respuestas expuestas en la actividad } \\
14 .\end{array}$ & \\
\hline 17 & $\begin{array}{l}\text { Investigación } \\
\text { sobre el } \\
\text { entorno. } \\
\text { AC+AR+C. }\end{array}$ & $\begin{array}{l}\text { Recoger, analizar e interpretar } \\
\text { información sobre cómo afecta } \\
\text { la situación actual provocada por } \\
\text { el CoviD-19 a Disneyland y qué } \\
\text { decisiones están tomando. Identificar } \\
\text { los factores del entorno que entran en } \\
\text { juego. Puesta en común y debate. }\end{array}$ & \\
\hline 18 & $\begin{array}{l}\text { Cierre de la } \\
\text { sesión. C. }\end{array}$ & Test final. & 5 min \\
\hline
\end{tabular}

\section{Cuestionario realizado al alumnado antes y después del CIMA}

Para la exploración de ideas previas y para el análisis de la evolución en el aprendizaje de los contenidos de la asignatura donde apliqué este CIMA, he usado un cuestionario con diseño pretest-postest (Guerra-Martín, 2014). El cuestionario se ha construido teniendo en cuenta las recomendaciones propuestas por Rivero y Porlán (2017) entre las que se incluyen: realizar preguntas abiertas, preguntar de forma indirecta y poner a los estudiantes ante situaciones hipotéticas. Con ello, traté de provocar respuestas espontáneas que me permitiesen explorar los modelos mentales de los estudiantes. Las preguntas que incluí en el cuestionario han sido las siguientes:

Pregunta 1. Si tuviésemos que descomponer a Ryanair en 'pedazos' que dieran lugar a diferentes subsistemas ¿Qué separación propones? Indica, según tu criterio, de qué se encargaría cada uno de ellos.

Pregunta 2. ¿Cuáles dirías que son los elementos que podrían darse, en la cultura empresarial de Ryanair? Razona tu respuesta.

Ciclos de Mejora en el Aula (2020). Experiencias de Innovación Docente de la US Esta obra se distribuye con la licencia Creative Commons 
Pregunta 3. Imaginamos que somos parte de la alta dirección de Ryanair y estamos tratando de diseñar la estrategia de cara a los próximos tres años. ¿Cuáles son los factores del entorno general que deberíamos tener en cuenta? ¿Por qué?

Pregunta 4. Seguimos tratando de diseñar la estrategia de Ryanair, ahora miramos al entorno específico. ¿Cuáles son los factores que tendríamos en cuenta? ¿Cómo podría afectar cada uno de ellos a Ryanair?

\section{Aplicación del ciclo de mejora en el aula}

\section{Relato resumido de las sesiones}

El diario de las sesiones se presenta en la tabla 2. Aquí, he recogido tanto cómo fue el desarrollo de las actividades diseñadas, como actitudes, reflexiones y emociones vividas en clase durante el desarrollo del CIMA.

Tabla 2. Relato resumido de las sesiones

La asistencia a clase fue de entre 10 y 15estudiantes en las sesiones impartidas de forma presencial.

\begin{tabular}{|l|l|l|}
\hline \multicolumn{3}{|c|}{ Sesión 1 } \\
\hline Act. & \multicolumn{1}{|c|}{ Desarrollo y reflexión } & $\begin{array}{c}\text { Tiempo } \\
\text { estimado/ } \\
\text { real }\end{array}$ \\
\hline 1 & $\begin{array}{l}\text { Esta actividad se realiza tal cual lo previsto. Parece } \\
\text { llamar la atención de los estudiantes preguntarles si es } \\
\text { importante estudiar lo que estudiamos y por qué. De esta } \\
\text { forma consigo crear una disposición previa del alumnado } \\
\text { que favorece el desarrollo de las siguientes actividades. } \\
\text { Los estudiantes se toman en serio la actividad, hay } \\
\text { silencio, se concentran. La sensación es positiva, el } \\
\text { debate es interesante y da lugar a una buena "nube de } \\
\text { conocimiento" que tenemos que ir ampliando y dándole } \\
\text { forma. }\end{array}$ \\
\hline
\end{tabular}

Ciclos de Mejora en el Aula (2020). Experiencias de Innovación Docente de la US Esta obra se distribuye con la licencia Creative Commons 


\begin{tabular}{|c|c|c|}
\hline 2 & $\begin{array}{l}\text { La explicación teórica participativa resulta muy eficaz para } \\
\text { que los alumnos y alumnas asimilen los contenidos, creo } \\
\text { que la actividad anterior los ha despertado lo que influye } \\
\text { en el dinamismo obtenido en esta actividad. Sin embargo, } \\
\text { la nueva forma de "estar en clase" nos hace dificil el } \\
\text { debate, no se escuchan, se interrumpen y hay silencios. }\end{array}$ & $\begin{array}{l}15 \min / 20 \\
\min \end{array}$ \\
\hline 3 & $\begin{array}{l}\text { Actividad muy interesante para identificar los } \\
\text { errores conceptuales en los que incurren o en los } \\
\text { que podrían incurrir los estudiantes. Ocurrió que, el } \\
\text { alumnado, al escuchar diferentes respuestas de sus } \\
\text { compañeros, son realmente conscientes de porqué } \\
\text { algunas respuestas no son válidas y su justificación y } \\
\text { cuáles dentro de las que sí lo son, se ajustan más a la } \\
\text { realidad. En mi opinión, la pizarra como lienzo, siempre } \\
\text { es una ayuda para aclarar ideas. Sin embargo, hubiera } \\
\text { imaginado un conocimiento de la materia mucho mayor } \\
\text { del que muestran. }\end{array}$ & $\begin{array}{l}15 \mathrm{~min} / 20 \\
\min \end{array}$ \\
\hline 4 & $\begin{array}{l}\text { Con esta actividad pretende fortalecer uno de los } \\
\text { contenidos que se ha visto dificultoso en otros cursos. } \\
\text { Desde mi punto de vista, al adquirir el alumno o } \\
\text { alumna un papel protagonista en la situación que se } \\
\text { presenta, observo, se consigue vencer este obstáculo. Esta } \\
\text { actividad obtiene muy buena acogida. Tengo la sensación } \\
\text { de que aclara este punto. }\end{array}$ & $\begin{array}{l}15 \min / 10 \\
\min \end{array}$ \\
\hline 5 & $\begin{array}{l}\text { En mi opinión, esta actividad no obtiene el resultado } \\
\text { esperado. Los noto desorientados y cansados tras toda } \\
\text { la sesión. Además, es realizada con bastante celeridad ya } \\
\text { que llega la hora de fin de la sesión. }\end{array}$ & $\begin{array}{l}15 \min / 5 \\
\min \end{array}$ \\
\hline \multicolumn{3}{|c|}{ Sesión 2} \\
\hline 6 & $\begin{array}{l}\text { Esta actividad esclarecedora para mí, ya que, puedo } \\
\text { conocer qué piensan y cómo han llegado a pensar de esta } \\
\text { forma. Además, para ellos, conocer las respuestas de sus } \\
\text { compañeros, identificar los errores de forma conjunta, } \\
\text { analizar y autoanalizarse buscando el porqué de las } \\
\text { respuestas, me comentan, que es una tarea esencial y } \\
\text { beneficiosa para avanzar en los contenidos de la materia. }\end{array}$ & $\begin{array}{l}20 \min / 25 \\
\min \end{array}$ \\
\hline 7 & $\begin{array}{l}\text { A pesar de que, en la primera sesión, la proyección } \\
\text { del mapa no dio los resultados esperados, esta vez es } \\
\text { diferente. Los estudiantes pueden ver cada parte dentro } \\
\text { de un todo, que de otra forma no hubiese sido posible. } \\
\text { Con sus preguntas siento que aumenta el interés por la } \\
\text { asignatura. }\end{array}$ & $\begin{array}{l}10 \min / 5 \\
\min \end{array}$ \\
\hline
\end{tabular}

Ciclos de Mejora en el Aula (2020). Experiencias de Innovación Docente de la US Esta obra se distribuye con la licencia Creative Commons Reconocimiento-NoComercial-SinObraDerivada Internacional (CC BY-NC-ND 4.0.) 


\begin{tabular}{|c|c|c|}
\hline 8 & $\begin{array}{l}\text { Consigo su atención y aumentar su interés ya que al } \\
\text { plasmar sus respuestas en la pizarra no las cuestiono. Sin } \\
\text { embargo, son ellos mismos los que llaman mi atención } \\
\text { para mostrar su acuerdo o desacuerdo con lo que se está } \\
\text { escribiendo en la pizarra. Apunto todo lo que me dicen, } \\
\text { pero no rectifico nada. En esta sesión, observo que los } \\
\text { estudiantes están muy receptivos. }\end{array}$ & $\begin{array}{l}20 \min / 10 \\
\min \end{array}$ \\
\hline 9 & \multirow[b]{2}{*}{$\begin{array}{l}\text { Estas dos actividades se acabaron mezclando, ya } \\
\text { que, mientras abordamos los contenidos sobre los } \\
\text { subsistemas empresariales, los alumnos y alumnas } \\
\text { participan poniendo ejemplos del caso o con dudas en } \\
\text { referencia a este. Aunque la previsión era abordar de } \\
\text { manera separada estas dos actividades, la interrelación } \\
\text { entre ambas, se desarrolla con la mayor naturalidad. } \\
\text { Esto, refleja el espíritu crítico despertado en el alumnado, } \\
\text { asunto que me alegra de sobremanera. Durante toda } \\
\text { la sesión planteo modificaciones a sus respuestas y } \\
\text { diferentes perspectivas. } \\
\text { Aun así, propongo una fase final donde revisamos } \\
\text { explícitamente cómo hemos ido corrigiendo las } \\
\text { respuestas previas. Adquiero un rol de guía y apoyo a } \\
\text { sus argumentaciones. Además, es en este momento } \\
\text { donde presentan que han descubierto sobre cómo está } \\
\text { actuando actualmente Disney respecto a las cuestiones } \\
\text { planteadas, mientras se ve envuelta en la crisis provocada } \\
\text { por el coviD-19. }\end{array}$} & \multirow{2}{*}{$\begin{array}{l}30 \min + \\
20 \min / 70 \\
\min \end{array}$} \\
\hline 10 & & \\
\hline 11 & $\begin{array}{l}\text { Por el amplio debate generado en la actividad anterior, } \\
\text { esta actividad no se terminó como se esperaba. Hacemos } \\
\text { el Kahoot. Analizamos las respuestas de manera muy } \\
\text { apresurada. Personalmente me permite detectar dónde } \\
\text { están los mayores obstáculos. }\end{array}$ & $\begin{array}{l}20 \min / 10 \\
\min \end{array}$ \\
\hline \multicolumn{3}{|c|}{ Sesión 3} \\
\hline \multicolumn{3}{|c|}{$\begin{array}{l}\text { Trabajan de forma autónoma mediante la plataforma de enseñanza virtual, } \\
\text { usando la herramienta Blackborad Collaborate Ultra. Controlo la participación } \\
\text { por la entrega de las tareas planificadas. }\end{array}$} \\
\hline \multicolumn{3}{|c|}{ Sesión 4} \\
\hline
\end{tabular}

Ciclos de Mejora en el Aula (2020). Experiencias de Innovación Docente de la US Esta obra se distribuye con la licencia Creative Commons 
Esta sesión se realiza de manera síncrona mediante la plataforma de enseñanza virtual. A pesar de no tener ningún tipo de problema técnico en su desarrollo, siento que pierdo información sobre su aprendizaje. Por una parte, la comunicación virtual se desarrolla de forma menos ágil de lo que se viene observando en clase. Por otro lado, al no verlos personalmente, no identifico cuándo se muestran perdidos o desconectados, aunque continuamente voy haciendo preguntas para responder por el chat e identificar de cierta forma si estamos siguiendo la clase. Considero la comunicación no verbal esencial en el proceso de enseñanza- aprendizaje y con el paso a enseñanza telemática la hemos perdido.

\begin{tabular}{|c|c|c|}
\hline 14 & $\begin{array}{l}\text { Esta actividad es para mí todo un descubrimiento. } \\
\text { Comenzar la clase con una puesta en situación y con } \\
\text { la explicitación de sus ideas logro un cambio en el } \\
\text { comportamiento del alumnado de manera que partiendo } \\
\text { de la aceptación de los errores los motiva a participar. } \\
\text { Esta situación los incomoda, los fuerza a pensar y a } \\
\text { expresarlo. Cuando captas su atención empiezan a } \\
\text { activarse a desconectarse del entorno y a conectarse con } \\
\text { la materia. } \\
\text { Se van acostumbrando a empezar las sesiones con un } \\
\text { ¿dónde estamos y a dónde los dirigimos? Y con un ¿qué } \\
\text { pensábamos antes de esta sesión? Me parece una buena } \\
\text { práctica a mantener }\end{array}$ & $\begin{array}{l}20 \min / 15 \\
\min \end{array}$ \\
\hline 15 & \multirow{3}{*}{$\begin{array}{l}\text { Estas tres actividades se han visto imbricadas. Sin } \\
\text { haber sido planificada, el resultado es enriquecedor. } \\
\text { Es el alumnado el que han modificado la secuencia } \\
\text { de actividades de manera natural, con sus preguntas } \\
\text { y aportaciones. Considero, que cuando algo fluye hay } \\
\text { que dejarlo que ocurra por lo que no he cortado sus } \\
\text { intervenciones para abordarlas en un momento posterior, } \\
\text { sino que avanzamos juntos en los contenidos al mismo } \\
\text { tiempo que resolvemos el caso y planteamos situaciones } \\
\text { actuales y futuras. }\end{array}$} & \multirow{3}{*}{$\begin{array}{l}20 \min + \\
25 \min + \\
30 \min / 90 \\
\min \end{array}$} \\
\hline 16 & & \\
\hline 17 & & \\
\hline
\end{tabular}

\section{Evaluación del aprendizaje del alumnado}

El presente curso escolar está siendo inusual e incierto para todo el colectivo educativo, dentro de él, alumnado y profesorado Con relación al alumnado he podido percibir un alto grado de desorientación, que la enseñanza semipresencial, en mi opinión, ha alimentado. Los estudiantes que han participado en este CIMA son de primer curso, recién aterrizados a la Universidad de Sevilla después de 
más de seis meses sin pisar las aulas y con el reto de la semi presencialidad, los cambios en la rotación de la asistencia, el cumplimiento de los protocolos sanitarios y el correcto seguimiento de las asignaturas. Todo esto ha provocado, momentos de desánimo, agobio y desinterés. Respecto al profesorado, lidiar con protocolos cambiantes y el manejo de las diversas emociones y situaciones de los alumnos y alumnas nos suponen una problemática añadida a la correcta realización de nuestra tarea docente.

En referencia a los cuestionarios inicial y final, he recogido 13 cuestionarios iniciales y 8 finales. Sin embargo, son 6 las personas que han cumplimentado ambos cuestionarios por la no asistencia de las mismas personas a todas las sesiones. Esto puede deberse al cambio forzoso de docencia presencial a telemática, a la falta de interés que puede provocar que la realización de esta tarea no tenga calificación alguna o a los exámenes parciales de otras asignaturas que han tenido lugar durante la realización del CIMA.

El análisis de las respuestas a los cuestionarios me ha permitido identificar respuestas similares y obstáculos presentes en el aprendizaje de la materia. Así, he podido clasificar las respuestas en varias categorías (tabla 3). El análisis del contenido de los cuestionarios iniciales me ha resultado sorprendente, imaginaba un grado de conocimiento más alto de los contenidos de la materia por parte de los estudiantes.

Ciclos de Mejora en el Aula (2020). Experiencias de Innovación Docente de la US Esta obra se distribuye con la licencia Creative Commons 
Tabla 3. Categorías de progresión de los conocimientos

\begin{tabular}{|c|c|c|c|c|}
\hline & Pregunta 1 & Pregunta 2 & Pregunta 3 & Pregunta 4 \\
\hline D & $\begin{array}{l}\text { No contesta o } \\
\text { contesta algo } \\
\text { que no se ha } \\
\text { preguntado }\end{array}$ & $\begin{array}{l}\text { No contesta o } \\
\text { contesta algo } \\
\text { que no se ha } \\
\text { preguntado. } \\
\text { Confunde cultura } \\
\text { empresarial con } \\
\text { cultura de una } \\
\text { sociedad o un } \\
\text { territorio; o con } \\
\text { un objetivo de la } \\
\text { organización. }\end{array}$ & $\begin{array}{l}\text { No contesta o } \\
\text { contesta algo } \\
\text { que no se ha } \\
\text { preguntado }\end{array}$ & $\begin{array}{l}\text { No contesta o } \\
\text { contesta algo } \\
\text { que no se ha } \\
\text { preguntado }\end{array}$ \\
\hline C & $\begin{array}{l}\text { Propone una } \\
\text { clasificación, } \\
\text { pero no } \\
\text { cumple con las } \\
\text { características de } \\
\text { un sistema }\end{array}$ & $\begin{array}{l}\text { Identifica } \\
\text { características } \\
\text { de la cultura, } \\
\text { aunque no } \\
\text { elementos }\end{array}$ & $\begin{array}{l}\text { Confunde } \\
\text { factores } \\
\text { internos de una } \\
\text { organización con } \\
\text { factores de su } \\
\text { entorno }\end{array}$ & $\begin{array}{l}\text { Confunde } \\
\text { factores } \\
\text { internos de una } \\
\text { organización con } \\
\text { factores de su } \\
\text { entorno }\end{array}$ \\
\hline B & $\begin{array}{l}\text { Enumera varios } \\
\text { o todos los } \\
\text { subsistemas } \\
\text { propuestos } \\
\text { para las } \\
\text { organizaciones } \\
\text { turísticas }\end{array}$ & $\begin{array}{l}\text { Enumera } \\
\text { elementos } \\
\text { que pueden } \\
\text { estar presentes } \\
\text { en la cultura } \\
\text { empresarial }\end{array}$ & $\begin{array}{l}\text { Mezcla factores } \\
\text { internos, del } \\
\text { entorno general } \\
\text { y del entorno } \\
\text { específico }\end{array}$ & $\begin{array}{l}\text { Mezcla factores } \\
\text { internos, del } \\
\text { entorno general } \\
\text { y del entorno } \\
\text { específico }\end{array}$ \\
\hline A & $\begin{array}{l}\text { Enumera y razona } \\
\text { la inclusión de } \\
\text { varios o todos } \\
\text { los subsistemas } \\
\text { propuestos } \\
\text { para las } \\
\text { organizaciones } \\
\text { turísticas } \\
\end{array}$ & $\begin{array}{l}\text { Enumera y razona } \\
\text { qué elementos } \\
\text { pueden estar } \\
\text { presentes } \\
\text { en la cultura } \\
\text { empresarial }\end{array}$ & $\begin{array}{l}\text { Enumera uno o } \\
\text { varios factores } \\
\text { del entorno } \\
\text { general con un } \\
\text { razonamiento } \\
\text { pobre o muy } \\
\text { pobre }\end{array}$ & $\begin{array}{l}\text { Enumera uno o } \\
\text { varios agentes } \\
\text { del entorno } \\
\text { específico sin } \\
\text { argumentar la } \\
\text { respuesta o con } \\
\text { un razonamiento } \\
\text { muy pobre }\end{array}$ \\
\hline$A^{+}$ & & & $\begin{array}{l}\text { Enumera y razona } \\
\text { factores del } \\
\text { entorno general } \\
\text { de manera bien } \\
\text { argumentada }\end{array}$ & $\begin{array}{l}\text { Enumera uno o } \\
\text { varios agentes } \\
\text { del entorno } \\
\text { específico } \\
\text { argumentando } \\
\text { profundamente } \\
\text { su respuesta }\end{array}$ \\
\hline
\end{tabular}

Ciclos de Mejora en el Aula (2020). Experiencias de Innovación Docente de la US Esta obra se distribuye con la licencia Creative Commons 
Para la evaluación inicial-final, he otorgado a cada pregunta dada en los cuestionarios de los estudiantes un nivel concreto. Gracias a ello he podido construir la tabla de evolución por alumno o alumna (tabla 4) y las escaleras de aprendizaje de cada pregunta (figura 3).

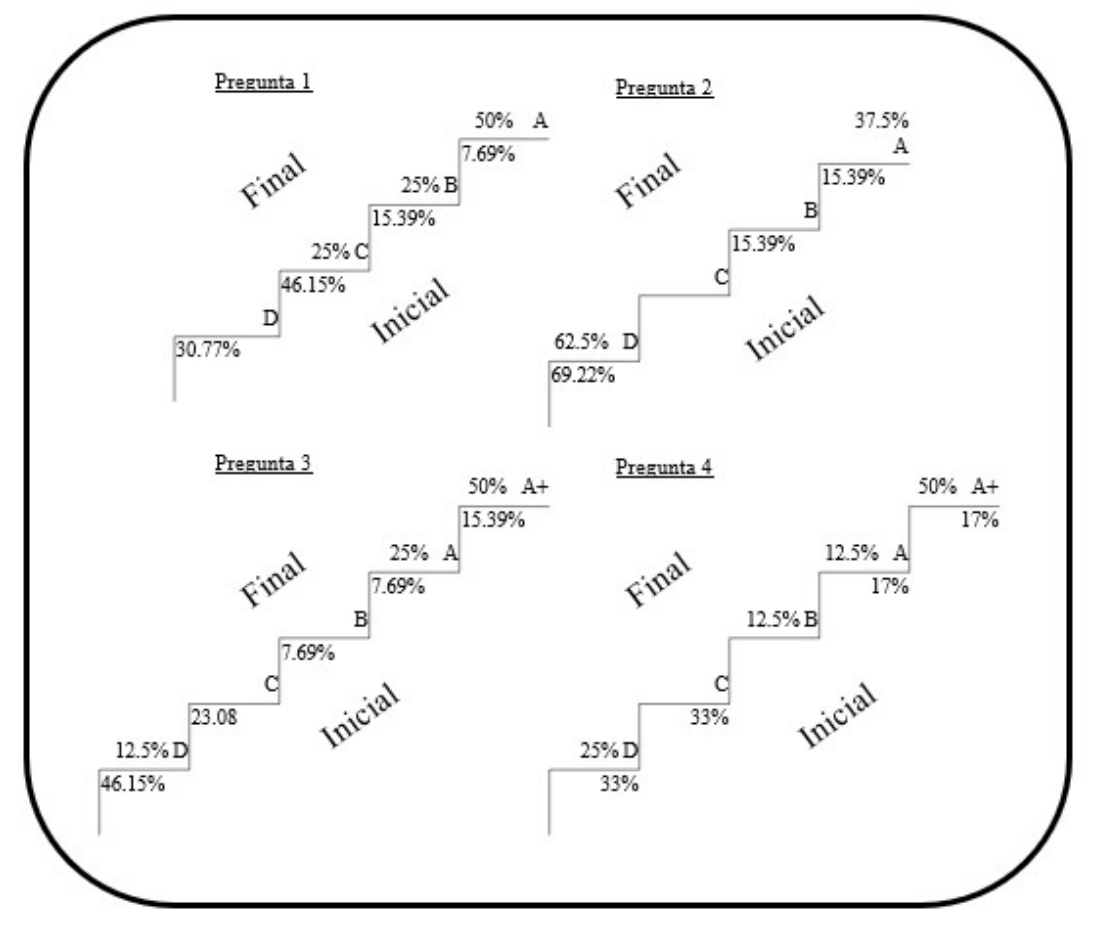

Figura 3. Escaleras de aprendizaje inicial- final por pregunta.

En relación con las escaleras de aprendizaje, es la pregunta 2 donde menos avance se ha logrado y la 3 en la que ha habido un mayor progreso. Tanto en la pregunta 3 como en la 4 esta evolución hasta llegar a alcanzar los escalones se ha conseguido por la identificación del obstáculo, la confusión entre qué es parte de la organización y qué pertenece al entorno. En la pregunta número 1 el mayor obstáculo se encontraba en la definición de sistema, una vez asimilaron que un sistema no era simplemente un conjunto de personas pudieron pasar a niveles superiores.

Ciclos de Mejora en el Aula (2020). Experiencias de Innovación Docente de la US Esta obra se distribuye con la licencia Creative Commons 
Tabla 4. Análisis comparativo de los cuestionarios por estudiante

\begin{tabular}{|l|l|l|l|l|l|l|l|l|l|}
\hline \multirow{2}{*}{ Estudiante } & \multicolumn{2}{l|}{ Pregunta 1 } & \multicolumn{2}{l|}{ Pregunta 2 } & \multicolumn{2}{l|}{ Pregunta 3 } & \multicolumn{2}{l|}{ Pregunta 4 } \\
\cline { 2 - 10 } & Inicial & Final & Inicial & Final & Inicial & Final & Inicial & Final \\
\hline 1 & A & A & A & A & A+ & A+ & A+ & $A^{+}$ \\
\hline 2 & C & C & D & D & B & A+ & D & A $^{+}$ \\
\hline 3 & D & A & D & D & D & A & D & C \\
\hline 4 & C & A & D & A & D & A+ & C & A \\
\hline 5 & C & A & B & A & A & A+ & A & A+ \\
\hline 6 & C & B & D & D & B & A & C & $A^{+}$ \\
\hline
\end{tabular}

En general observo tras el análisis de la comparación por estudiante (tabla 4) una subida de nivel, 4 de los 6 han mejorado en al menos tres de las cuatro preguntas. Además, dos de los estudiantes ha logrado un salto de más de dos escalones en la mitad de las preguntas del cuestionario y un tercero, ha logrado subir de escalón en todas las preguntas.

La realización de los cuestionarios me ha resultado tremendamente útil para identificar cuáles eran los obstáculos a los que se enfrentaban los alumnos y las alumnas que le impedían llegar a escalones superiores. Por lo que con el énfasis puesto en superar estos obstáculos y haciéndoles ver y reflexionar sobre sus respuestas, considero, que para el alumnado esta actividad también les ha sido útil.

A pesar de los contratiempos sobrevenidos durante el diseño, desarrollo y evaluación del CIMA, puedo observar mediante el análisis de las respuestas a los cuestionarios, también de aquellas que no han completado uno de los dos, que, de manera global, se ha conseguido una mejora en el aprendizaje del alumnado.

Ciclos de Mejora en el Aula (2020). Experiencias de Innovación Docente de la US Esta obra se distribuye con la licencia Creative Commons 


\section{Evaluación del ciclo de mejora en el aula}

La evaluación del CIMA la he llevado a cabo a través de la observación y de la reflexión sobre las notas recogidas en el diario de clase. Así, he sido consciente del modelo metodológico que estaba usando, qué aspectos funcionaban y cuáles no. El análisis de las impresiones percibidas en clase, antes, durante y tras la implementación del CIMA, me ha permitido percatarme, por ejemplo, del aumento de la participación del alumnado en clase, así como, de la mejora en sus reflexiones y en la construcción de sus respuestas respecto a los contenidos abordados. Con la implementación del CIMA he logrado una mejora en varias áreas de mi práctica docente habitual que seguiré poniendo en práctica:

- Respecto al propio aprendizaje de los alumnos y alumnas, ha sido un descubrimiento acercarme a conocer sus modelos mentales a priori. Leer, mostrar y reflexionar con los estudiantes sobre las respuestas que dan a problemas que versan sobre contenidos no abordados, ha sido tremendamente enriquecedor. Por ello, estoy dispuesta a incluir la explicitación de ideas en cada bloque temático.

- Respecto a la forma de abordar los contenidos, he percibido como facilitador en el aprendizaje de estos la visión global que ha podido tener el alumnado con la proyección del mapa de contenidos. Para ellos, mostrarles la ruta completa en cada sesión para que vean dónde nos encontramos y hacia dónde vamos, así como las interrelaciones entre los contenidos creo que es positivo.

- En cuanto a la metodología, he percibido que un cambio en las actividades o en el orden en las que las desarrollamos usualmente mantiene el interés del alumno o alumna a seguir la clase por la incertidumbre que despierta.

Ciclos de Mejora en el Aula (2020). Experiencias de Innovación Docente de la US Esta obra se distribuye con la licencia Creative Commons 
- Respecto a la evaluación, los cuestionarios iniciales me han resultado muy útiles para detectar obstáculos. Sin embargo, los cuestionarios finales no lo han sido tanto por la falta de respuestas. Esto me lleva a pensar que en futuras ocasiones es necesario obtener un compromiso expreso del alumnado. Por otro lado, pretendo incorporar el diario de las sesiones como elemento de evaluación en mi práctica docente habitual. Me propongo la creación de un banco de actividades con la descripción de qué pasó en clase cuando se llevó a cabo.

En resumen, el Curso General de Docencia Universitaria ha resultado ser una experiencia fructífera. Me ha proporcionado autoconocimiento de mi práctica docente habitual, instrumentos de mejora y ejemplos reales de buenas prácticas en un contexto turbulento e incierto. Todo esto, posibilita una mejora en el proceso enseñanza- aprendizaje que podré llevar conmigo a cualquier asignatura que imparta en el futuro.

Ciclos de Mejora en el Aula (2020). Experiencias de Innovación Docente de la US Esta obra se distribuye con la licencia Creative Commons 
Palabras clave: Empresas y Organizaciones Turísticas, Grado en Turismo, docencia universitaria, experimentación docente universitaria.

Keywords: Tourism Companies and Organizations, Tourism Degree, University Teaching, University Teaching Experiments.

\section{Referencias bibliográficas}

Bain, K. (2004). What the best college teachers do. Cambridge, MA: Harvard University Press. (Trad. cast.: Lo que hacen los mejores profesores universitarios. Valencia: Publicaciones de la Universidad de Valencia, 2007. 2a edición).

De Alba, N. y Porlán, R (2017). La metodología de enseñanza. En: R. Porlán ('Coord.), Enseñanza universitaria cómo mejorarla (pp-37-53). Madrid: Morata.

Guerra-Martín, M.D, (2014). Tutoring as a way of achieving employability for nursing students at the University of Seville. Procedia- Social and Behavioral Sciences, 139, 479-486.

Finkel, D. (2000). Teaching with your mouth shut. Portsmouth, NH: Heinemann Boynton/Cook. (Trad. cast.: Dar clase con la boca cerrada. Valencia: Publicaciones de la Universidad de Valencia, 2008).

Pedregoso-Mateos, B. (2018). Del modelo didáctico práctico al modelo basado en el principio de investigación. Aplicación a la asignatura Typologies of Tourism Areas del grado de Turismo. En E. Navarro-Medina y R. Porlán, Jornadas de Formación e Innovación Docente del Profesorado (pp. 500-516). Sevilla: Editorial Universidad de Sevilla.

Porlán, R. (2020). El cambio de la enseñanza y el aprendizaje en tiempos de pandemia. Revista de Educación Ambiental y Sostenibilidad, 2(1), 1502.

Rivero, A. y Porlán, R (2017). La evaluación en la enseñanza universitaria. En R. Porlán (Coord.), Enseñanza universitaria cómo mejorarla (pp-73-91). Madrid: Morata.

Rosa-Díaz, I.M. (2020). Experiencia de un Ciclo de Mejora en el Aula en el ámbito del marketing financiero. En E. Navarro-Medina y R. Porlán (Coord.), Ciclos de mejora en el aula. Año 2019. Experiencias de innovación docente

Ciclos de Mejora en el Aula (2020). Experiencias de Innovación Docente de la US Esta obra se distribuye con la licencia Creative Commons 
de la Universidad de Sevilla (pp. 313-335). Sevilla, España: Editorial de la Universidad de Sevilla.

Zerva, A. (2020). Implementación de un ciclo de mejora en el aula de la asignatura Francés Turístico. En E. Navarro-Medina y R. Porlán (Coord.), Ciclos de mejora en el aula. Año 2019. Experiencias de innovación docente de la Universidad de Sevilla (pp. 313-335). Sevilla, España: Editorial de la Universidad de Sevilla.

Ciclos de Mejora en el Aula (2020). Experiencias de Innovación Docente de la US Esta obra se distribuye con la licencia Creative Commons 